\title{
Anti-allergy medicine tranilast inhibits IL-33 production by macrophages with concomitant suppression of Akt activity
}

\author{
Sachiko Hiraide, Yoshiki Yanagawa, Kenji lizuka
}

Department of Pharmacology, School of Pharmaceutical Sciences, Health Sciences University of Hokkaido, Japan

Background: Tranilast is an anti-allergy medication that inhibits the release of chemical mediators such as histamine. However, the mechanisms underlying its anti-allergy effects are not fully understood. Interleukin (IL)-33, a novel member of the IL-1 cytokine family, promotes Thelper type 2 immune responses and plays a pathogenic role in allergic disorders. In the present study, we examined the effects of tranilast on IL-33 production by RAW264.7 macrophages and bonemarrow derived macrophages were used.

Methods: IL-33 protein was quantitated by ELISA. IL-33 mRNA level was determined by quantitative RT-PCR. Cell viability was evaluated by MTT assay. Phosphorylation of Akt, MAPKs, and NF-kappa B was analyzed by immunoblotting.

Results: Lipopolysaccharide (LPS) increased both IL-33 mRNA expression and IL-33 protein synthesis. Tranilast significantly inhibited LPS-induced IL-33 protein production by RAW264.7 macrophages in a dose-dependent manner; these same effects were observed on IL-33 mRNA levels in RAW264.7 macrophages and bone-marrow derived macrophages. Tranilast had no effect on the viability of RAW264.7 macrophages. LPS markedly activated Akt in RAW264.7 macrophages, whereas tranilast suppressed LPS-induced Akt activation. The effect of tranilast on Akt activation appeared to be responsible for the decrease in IL-33 production.

Conclusion: Our present findings suggest that the inhibition of IL-33 production by tranilast might contribute to the antiallergy effects of this medication. 